\title{
Conflictos socioambientales, emociones y empoderamiento: la resistencia contra la presa de San Nicolás en México
}

\author{
ALICE POMA Y TOMMASO GRAVANTE
}

Pretendemos mostrar el proceso de transformación de conciencia y de conducta experimentado por los miembros de una comunidad local que se opuso a la construcción de una presa que inundaría su territorio. Presentaremos algunos resultados, basados en el análisis de un conflicto, acerca de la dinámica de la protesta y las emociones como factores que influyen en la motivación para la acción y el proceso de transformación de conciencia y conducta. También mostraremos cómo cambia la relación entre la población local y su territorio. Por último, evidenciaremos algunas prácticas surgidas de la experiencia de conflicto.

PALABRAS CLAVE: luchas por la defensa del territorio, presas, emociones, transformación de conciencia y conducta, empoderamiento

Social Environmental Conflicts, Emotion and empowerment: The Resistance against the San Nicolás' Dam in Mexico

Alice Poma

Laboratorio de Análisis de Organizaciones y Movimientos Sociales, Centro de Investigaciones Interdisciplinarias en Ciencias

y Humanidades, Universidad Nacional Autónoma de México, Ciudad de México, México alicepoma@gmail.com

TOMMASO GRAVANTE

Laboratorio de Análisis de Organizaciones y Movimientos Sociales, Centro de Investigaciones Interdisciplinarias en Ciencias y Humanidades, Universidad Nacional Autónoma de México, Ciudad de México, México t.gravante@gmail.com
We describe the process of transformation of consciousness and behaviour experienced by mobilized members of a local community who opposed to the building of a dam that threatened to inundate their territory. We will present some results, based on analysis of a specific case of conflict, about the dynamic of protest and emotions as factors influencing both motivation for action and the process of transformation of consciousness and behaviour. We will also show how the relationship between local people and their territory changed. Finally, we will point some practices that emerged from the experience of conflict.

KEYWORDS: struggles in defense of the territory, dams, emotions, transformation of consciousness and behaviour, empowerment 


\section{Introducción}

as actividades de protesta de la gente común "han sido a menudo trivializa-

das, ignoradas y vistas como acciones interesadas, particularistas y parroquianas" pero más bien "revelan un mundo más amplio de poder y resistencia, [y] en cierta medida terminan por desafiar las relaciones sociales de poder" (Krauss, 1993: 248). A partir de esta premisa, en este artículo queremos presentar un análisis de la resistencia contra la construcción de la presa de San Nicolás, en Jalisco, México, y pondremos en evidencia cómo esta lucha para preservar la existencia del pueblo y la comunidad provocó un cambio profundo en las personas. Como escribieron las investigadoras Fedi y Mannarini:

La movilización, proponiendo modelos alternativos de desarrollo del territorio y dando vida a procesos de integración tanto dentro de las comunidades locales como entre éstas y la sociedad, vuelve a la ciudadanía más activa y consciente de sus potencialidades y de los recursos que tiene a disposición, sosteniendo así un proceso de empowerment o de desarrollo de comunidad, y puede hasta contribuir a la reconstrucción del "sentimiento" de comunidad (2008: 11-12).

El objetivo de nuestra investigación es analizar el cambio que experimenta la gente en "los breves momentos en los que está despierta por la indignación": “cuando [los grupos] estén preparados a desobedecer a las autoridades, a los que normalmente los aplastan, estos breves momentos en los que [...] los de abajo ejercen alguna fuerza contra el Estado" (Piven y Cloward, 1977: x), en ese instante, hay una transformación "tanto de conciencia como de conducta" (Piven y Cloward, 1977: 3) producida por la experiencia de la protesta. Como Jasper ha mostrado en sus investigaciones, "una vez que una persona empieza a participar está sujeta a nuevos procesos sociales que ayudan a dar formas a emociones, moral y cognición" (1997: 185). Como veremos en nuestros análisis, esto contribuye a reelaborar ideas, 
creencias y valores. Esa transformación es el resultado de procesos cognitivo-emocionales y es en sí misma un proceso "que no pasa tanto por influir sobre los responsables políticos, como por la transformación respecto a la manera de vivir juntos a partir de alternativas concretas que pongan en práctica los valores del movimiento y una reafirmación de las formas de sociabilidad locales" (Pleyers, 2009: 145).

Con base en el estudio de la dimensión cultural de la protesta, que comprende creencias cognitivas, respuestas emocionales y evaluaciones morales (Jasper, 1997), propondremos una lectura crítica de algunos aspectos que consideramos importantes al analizar los procesos de resistencia desde la perspectiva de los afectados. Con este trabajo queremos contribuir a la comprensión de los procesos sociales y culturales que se desencadenan en las luchas por la defensa del territorio.

\section{Metodología}

Las herramientas metodológicas empleadas en la investigación han sido las entrevistas en profundidad y semiestructuradas realizadas a habitantes del territorio afectado por la construcción de la presa de San Nicolás, que se autoorganizaron para defenderse. ${ }^{1}$ Los actores que hemos elegido para las entrevistas son: 1) habitantes de San Gaspar de los Reyes y Teocaltiche, que se autoorganizaron en el Comité Pro San Gaspar, y 2) habitantes de San Gaspar, Teocaltiche y ranchos cercanos que participaron en el conflicto sin ser miembros del comité. El análisis se basa en la experiencia de personas no vinculadas a ninguna organización política o no gubernamental, sino que defendieron su pueblo y tierras, con una relación directa tanto material como emocional con el territorio amenazado y han participado en la experiencia de resistencia sin tener experiencia previa de lucha. No obstante, para la compresión contextual del caso, hemos acudido a periodistas locales que colaboraron en el conflicto o conocían el contexto y los acontecimientos.

En palabras de Mairal, Bergua y Puyal, "nuestro objetivo, entre otros, ha sido mostrar un punto de vista, el de las partes, la más crítica y opositora, ya que hasta ahora se ha venido considerando perjudicada” (1997: 8). Como escribió Donatella Della Porta, "centrarse en el punto de vista de los activistas no es señal de parcialidad, sino una elección metodológica necesaria para comprender el por qué de sus acciones y poderlas interpretar" (2008: 21). Al centro del análisis ya no está el movimiento como actor social, sino las personas que luchan, que "siempre habían estado allí pero no teníamos ojos para verlas ni oídos para escucharlas" (Regalado, 2012: 170). Así, analizamos los procesos por medio de los cuales la protesta influye y cambia a los sujetos.

Como afirma Holloway (2011), las pequeñas experiencias cotidianas de rebeldía, aunque sean contradictorias y experimentales, son momentos de ruptura. En términos metafóricos, son grietas en el sistema, porque en ellas se experimenta un otro-hacer, un vivir a otro ritmo, que tiene un gran potencial de cambio. Aunque no todas las grietas sean del mismo tamaño o profundidad ni produzcan el mismo efecto en el sistema, el problema hasta ahora es haber ignorado las líneas de continuidad entre las más diversas experiencias alrededor del mundo. Hablar de las grietas significa, en palabras del autor, "hallar formas de hacer visible y fortalecer estas líneas de continuidad que, a menudo, están sumergidas"

1 El caso de estudio forma parte de la investigación doctoral de Poma (2013). Cuenta con un total de 21 entrevistas grabadas digitalmente y trascritas, cuya duración varía de 45 minutos a dos horas. Las de mayor extensión son excepcionales. Para garantizar el anonimato de los participantes, los distinguimos con siglas. El lector encontrará al final de la bibliografía una relación que indica el sexo del entrevistado, su papel en el conflicto -organizador o participante-y la fecha de la entrevista. Cualquier información adicional hubiera podido delatar la identidad de la persona. 
(Holloway, 2011: 40). Añadimos que los sujetos no se pueden comprender sin considerar la rutina y la cotidianidad, profundamente relacionadas con el cambio social porque, como veremos, "la experiencia de todos los días de la gente [...] contribuye a construir el sentimiento de injusticia, establece la mesura de sus demandas y pone en evidencia los objetivos de su rabia" (Piven y Cloward, 1977: 20-21).

El paso sucesivo es reconocer el peso de las decisiones personales más allá de la dimensión personal y privada porque "la gente decide qué hacer y estas decisiones tienen importantes efectos" (Jasper, 1997: XII) y "los miles de decisiones conscientes de este tipo son tan importantes, si no más, que los actos de los políticos para explicar los modelos del cambio social" (Thompson, 1993: 74). Pasamos ahora al caso de la resistencia contra la presa de San Nicolás en San Gaspar de los Reyes, Jalisco, México.

\section{La lucha contra la presa de San Nicolás, Los Altos de Jalisco, Jalisco, México}

Al llegar a San Gaspar de los Reyes, en Los Altos de Jalisco, México, parece que no queda rastro de lo que fue una experiencia de resistencia ejemplar, de una población que se volcó a la lucha por la defensa de su pueblo y sus raíces, que superó la idea de que "con el gobierno no se puede". Sin embargo, es suficiente hablar con las personas que participaron en esa experiencia para revivirla, sentir lo que sentían y ver lo que un ejercicio como éste dejó en los que lo vivieron.

La presa de San Nicolás, ${ }^{2}$ que inundaría los pueblos de San Gaspar de los Reyes y San Nicolás de las Flores, ambos en el municipio de Jalostotitlán, y muchos ranchos y tierras fértiles del municipio de Teocaltiche, era uno de los muchos proyectos para represar el río verde que se sumaban a la historia y memoria de los habitantes de estas tierras (Cabrales, 1993; Casillas, 1999; 2002; Hernández y
Casillas, 2006; 2008). Al principio, la población no lo creía porque, según contaban los entrevistados, habían vivido décadas con la amenaza de una presa que nunca se había construido.

Acerca del uso del agua, el proyecto preveía el trasvase del agua al cercano estado de Guanajuato. Es interesante destacar que los afectados reconocen la importancia del agua para el desarrollo de la sociedad. ${ }^{3}$ Por esa razón, se informan sobre las características de los proyectos y las alternativas que según su criterio, en primer lugar, no tienen que afectar a otros pueblos, ${ }^{4} \mathrm{y}$ en segundo, tienen que respetar el medio ambiente. La existencia de alternativas viables, muchas veces más económicas y que no afecten a personas alimenta los sentimientos de ultraje e injusticia que sienten los afectados. Al respecto, y como parte de los procesos de trasformación de conciencia y conducta que analizaremos, en todos los casos, los afectados se convierten en defensores de sus derechos y denuncian la naturaleza política de los proyectos, que esconden intereses personales de los políticos implicados, más que motivaciones técnicas reales o de interés general. Es preciso añadir que la construcción de una presa

La cortina prevista era de 65 metros de altura, 8000 hectáreas de extensión, con una capacidad aproximada de 800 hectómetros cúbicos. Para un análisis del contexto del proyecto de la presa de San Nicolás, véase Hernández y Casillas (2006; 2008).

3 Los entrevistados afirmaron que en caso de no haber tenido alternativas, hubieran visto si el proyecto era imprescindible y de interés común, y hubieran estado dispuestos a encontrar una solución: "si no iba a haber de otra, el agua se ocupa, estoy consciente de que primero el aire y después el agua para vivir [...]. El agua la necesitamos todos y todos estamos de acuerdo de eso" (E.Sg.5b).

$4 \quad$ En el caso de San Nicolás, el proyecto alternativo que decidió llevar a cabo el gobierno federal -la presa del Zapotillo que inundará a Temacapulín, Acasico y Palmarejo, cuyo conflicto sigue activo en la actualidad - nunca fue el propuesto por el Comité Pro San Gaspar, cuya postura siempre fue defender opciones que no afectaran a otras poblaciones. 
tiene consecuencias en el reparto del agua, que al ser almacenada, es controlada por la empresa adjudicataria de la explotación o por el Estado, los cuales prohíben el acceso y uso a los habitantes de los lugares afectados. Esto agudiza el empobrecimiento de la zona y le quita a los pobladores cualquier poder de decisión y gestión sobre su territorio:

Sabemos que una presa, y de acuerdo con la experiencia que tuvimos, destruye ríos, arroyos, o sea, acaba, deseca muchas veces los lugares, porque el agua se aglutina en un solo punto y después es controlada... El campesino que tenía un arroyito, que tenía algo de dónde tomar, que tenía un pozo, un manto freático o algo, se acaba, se destruye (E.Sg.1).

Sobre la índole de estos proyectos, se confirma la tesis que defiende su carácter simbólico y político (Pearce, 1992; Ward, 2003; McCully 2004). De nuestro análisis emerge con claridad la idea de que la decisión de realizar los proyectos responde más a lógicas políticas que a planteamientos técnicos de política hidráulica:

Levanta demasiadas dudas el hecho de que el proyecto de El Zapotillo, presentado como emergente, se encontrara mejor documentado que el proyecto de San Gaspar. Todo eso nos lleva a suponer que el proyecto original era el presentado como emergente, mientras que estratégicamente se planteó el otro con la intención de desviar la atención y en caso de prosperar, levantar un sistema de represas interconectadas tendentes, como prioridad, al abasto de las ciudades (Hernández y Báez, 2006: 38-39).

Según los elementos que caracterizan a las obras, éstas afectan las tierras más fértiles y perjudican aún más a quienes viven del campo ${ }^{5}$ la ganadería, pues no sólo pierden su casa, sino también su sustento y su manera de vivir. El destino más común para estas personas es alguna ciudad, en la que se les ofrece trabajo en la construcción o en las industrias. A esto se añade la desigualdad en la afectación. Familias con numerosas propiedades pueden conseguir beneficios económicos gracias a la expropiación, que les permitirán vivir e invertir en otros lugares, mientras que personas y familias sin bienes son despojadas de lo poco que poseen sin la posibilidad de empezar de nuevo en otro sitio. Lo mismo vale para aquellas personas con estudios y oportunidades de insertarse en el mundo laboral urbano, mientras que, por su biografía o edad, otras no tendrán estas oportunidades y perderán su estilo de vida en el pueblo para engrosar las periferias urbanas en busca de un trabajo no cualificado. Todo eso sin hablar de las desigualdades e injusticias que ocurran a la hora de repartir las expropiaciones, pues no es raro que haya canales preferentes y corrupción, así como retrasos, con la consecuente pérdida de valor. En los pocos casos de pueblos reubicados, se pierden las estructuras sociales y culturales tradicionales, y la calidad de la vivienda nueva por lo general es inferior a la que se deja. Las consecuencias no se presentan una vez que concluye el proyecto, sino que en todos los casos que hemos estudiado, hemos comprobado que la amenaza de construcción de la presa había llevado a la dejadez y desatención del pueblo. Como los habitantes no saben si se quedarán en el pueblo, no invierten en sus casas y tierras, la comunidad no invierte en infraestructuras, y poco a poco el pueblo es abandonado:

Ya era como un pueblo fantasma, porque la gente ya no quería construir nada [...], la gente de aquí no había ningún modo de mantenerse, la gente ya no quería construir, duramos como dos años que la gente ya no hacía nada y la gente, pues, tiene que trabajar para mantenerse (E.Sg.5).

5

En el área estudiada se vive principalmente de la ganadería y del cultivo de maíz, frijol y alfalfa. 
Hablamos de una zona que podríamos definir como periférica respecto a los centros económicos y de poder. En San Gaspar de los Reyes, los ingresos económicos de las familias son las remesas de los parientes que están en Estados Unidos, ${ }^{6}$ seguidas por el trabajo en las granjas intensivas que ocupan la comarca y el cultivo de la tierra. Estas zonas dependen de las zonas urbanas más cercanas, en las que se encuentran los servicios, la oferta de trabajo, etc., y se consideran lugares "sacrificables" para el bienestar de otras zonas, ${ }^{7}$ ya sea para proporcionar agua para consumo humano, regadío o energía eléctrica. La estigmatización de pueblos y personas "de segunda categoría" es uno de los elementos que influyen en la transformación de conciencia y de conducta de los que se oponen a la obra hidráulica, que alimenta lo que Scott (2000) define como el "discurso oculto".

Esa característica de marginalidad es muy importante. Como observan Pedro Tomé y Andrés Fábregas (1999), ${ }^{8}$ el carácter periférico forja una serie de procesos endógenos que contribuyen a crear una cierta identidad. En el caso de San Gaspar, encontramos una cultura enraizada en valores y principios católicos profundos, en los que, por un lado, emerge un rechazo a todo lo que se refiere a la cultura de protesta, de izquierda, ecologista, etc., mientras que, por el otro, encontramos la importancia de la comunidad — como ellos mismos se definen-y la solidaridad, sobre todo en momentos de dificultad y con miembros de la propia comunidad, que los entrevistados comparan con una gran familia.

En este contexto, no sorprende que la intervención estatal sea percibida como una amenaza a la que hay que resistir. De hecho, los afectados hacen referencia a la herencia histórica de las guerras cristeras. Esa identidad histórica es relevante a la hora de reivindicar una posición antiestatal, pero también, como apuntaron algunos entrevistados, a la hora de confiar en algún poder, ya que la guerra terminó con un acuerdo entre el Estado y la Iglesia católica, que convirtió a los combatientes en bandidos y causó una mayor disgregación de la comunidad cuando muchos huyeron de sus pueblos.

Como afirma Mairal, la realización de un embalse presupone un antes y un después que cambia "sustancialmente las vidas de los individuos afectados por una parte y [altera] la estructura socio-espacial por otra", se convierte en "algo muy profundo en la experiencia vital colectiva” (1993: 185). Entre otras cosas, produce una transformación de conciencia y de conducta.

Para concluir con la presentación del caso, nos relataron que el conflicto empezó "como en todos los pueblos pequeños, con rumores” (E.Sg.9). La inquietud y la tenacidad de algunos habitantes de la zona afectada rompieron el silencio institucional para acceder a unas cuantas informaciones y avisar a los vecinos del peligro que se acercaba. En 2004, a finales de abril, se llevó a cabo la primera reunión organizada por el Comité Pro San Gaspar para informar a los vecinos y pensar cómo actuar. En menos de un mes, el pueblo estaba lleno de letreros de los afectados que expresaban el rechazo al proyecto. Los meses siguientes hubo reuniones, manifestaciones, etc.; fueron meses "duros, durísimos", "de dar muchos topes, muchas vueltas" (E.Sg.7). Los habitantes de la zona afectada, en particular los miembros del Comité, tuvieron que poner a un lado sus vidas para defender a su pueblo.

El conflicto, que en su manifestación pública más evidente duró poco más de un año, terminó

6 En la actualidad, casi la mitad de los habitantes del pueblo vive fuera.

7 Gaspar Mairal (1993) señala la polaridad territorial entre llanura y montaña. La primera aprovecha los recursos hídricos, mientras que la segunda sufre los impactos territoriales. De la misma manera podríamos hablar de campo y ciudad, interior y litoral, etcétera.

8 Proponen un estudio comparado de los procesos culturales en Los Altos de Jalisco, México, y la provincia de Ávila, España, con un marco teórico y metodológico basado en la ecología cultural y la etnografía. 
el 31 de mayo de 2005 con la declaración del secretario de Gobernación de Jalisco sobre la desestimación del proyecto. San Gaspar de los Reyes, San Nicolás de las Flores y todos los ranchos y comunidades de la zona estaban a salvo, al menos hasta la instrumentación de otro proyecto. El éxito de la protesta no puede entenderse sin la colaboración de actores externos, como el Movimiento Mexicano de Afectados por las Presas y en Defensa de los Ríos (Mapder), el Comité de Ausentes de San Gaspar en Acción (CASA), integrado por los hijos ausentes que residen en Estados Unidos, periodistas locales, algún político no comprometido con el proyecto y protagonistas de experiencias similares de otras partes del país, que "inyectaron valor" (E.Sg.5) a los habitantes de la comarca.

\section{Las emociones como factores explicativos}

Uno de los retos que nos hemos propuesto en el desarrollo de la investigación ha sido incorporar las emociones como factores explicativos del proceso de resistencia. Como afirman las investigadoras Fedi y Mannarini, "raramente ha sido analizado el significado simbólico y emotivo que la defensa del propio territorio supone" (2008: 11). El análisis de la experiencia de quienes luchan y sus emociones ayuda a entender mejor ciertas dinámicas propias de la protesta, ${ }^{9}$ por ejemplo, por qué la gente participa - tema estudiado con frecuencia en la literatura sobre movimientos sociales-, por qué abandona, qué relaciones se crean entre las personas o cómo la participación influye y cambia sus vidas. De hecho, el análisis de la dimensión emocional de la protesta permite comprender procesos como la transformación de conciencia y de conducta (Piven y Cloward, 1977), que se habían desarrollado sin incorporar las emociones. Estos autores escribieron en un periodo en el que los estudiosos de la protesta negaban las emociones, como recuerda Jasper (2014).
En los siguientes apartados nos centraremos en entender el papel del "apego al lugar". Se trata de un vínculo afectivo central en las experiencias de lucha para la defensa del territorio, porque es uno de los motivadores de la protesta que facilita la comprensión de lo que los afectados defienden y por lo que luchan. Concluiremos con un párrafo acerca de los cambios que observamos en quienes participaron en el conflicto.

\section{EL APEGO AL LUGAR ${ }^{10}$}

Definido como la "conexión cognitiva y emocional de un individuo a un escenario o ambiente particular" (Low y Altman, 1992: 165), el apego al lugar es un proceso emocional y cognitivo complejo que está recibiendo mucha atención por parte de los científicos sociales que estudian la relación entre las personas y los espacios y territorios que habitan. A pesar de que los autores que analizan el concepto reconocen que su desarrollo teórico es aún insuficiente (Giuliani, 2004; Manzo y Devine-Wright, 2014), muchos avances han tenido lugar en los últimos 20 años. En particular, el apego al lugar se ha aplicado al estudio de la gestión de los recursos naturales, de las energías alternativas, de los conflictos de las comunidades locales contra la instalación de proyectos, de los comportamientos proambientales, de la respuesta a los desastres y de los desplazamientos (Low y Altman, 1992; Devine-Wright, 2009; Manzo y Devine-Wright, 2014).

9 La literatura sociológica de los últimos 20 años ha demostrado, por ejemplo, que las emociones no sólo "tienen efectos significativos en los movimientos” (Gould, 2004: 162), sino que también "ayudan a explicar el origen, el desarrollo y el éxito o no del movimiento” (Jasper, 1998: 416-417).

10 Este concepto se ha desarrollado principalmente en la psicología ambiental y la sociología urbana. Para una revisión de la literatura en español, véase Hidalgo (1998). 
Low y Altman (1992: 165) propusieron una lectura cultural según la cual "el apego al lugar es la relación simbólica formada por personas que dan significados afectivos culturalmente compartidos a un espacio particular o porción de terreno que ofrece las bases para la comprensión individual y colectiva de la relación con el medio ambiente". En un trabajo más reciente, Hidalgo (1998) revisó otras tipologías de apego, como el infantil o adulto, y propone considerar el apego al lugar como:

Un lazo afectivo que una persona o animal forma entre él mismo y un determinado lugar, un lazo que le impulsa a permanecer junto a ese lugar en el espacio y en el tiempo. La característica más sobresaliente es la tendencia a lograr y mantener un cierto grado de proximidad al objeto de apego (1998: 51).
Al investigar qué papel jugó el apego al territorio en la resistencia contra la presa de San Nicolás, hemos apreciado que la intensidad emocional con la que se vinculan los entrevistados a su entorno natural y el sentimiento de apropiación de éste fueron elementos importantes en la motivación para la acción. Lo que animó a las personas entrevistadas a participar en la protesta fue, en primer lugar, el apego que sentían hacia el pueblo: "no queremos la presa, porque nosotros aquí nacimos, aquí nos criamos, aquí tenemos nuestro ombligo enterrado [...], aquí vivimos la juventud, la niñez, la vejez que estamos viviendo, [...] somos de aquí, que no queremos perder nuestra tierra" (E.Sg.10).

El pueblo es el lugar en el que residen los recuerdos: la fiesta del pueblo, los domingos en familia o con amigos, el trabajo diario en el campo. Todo

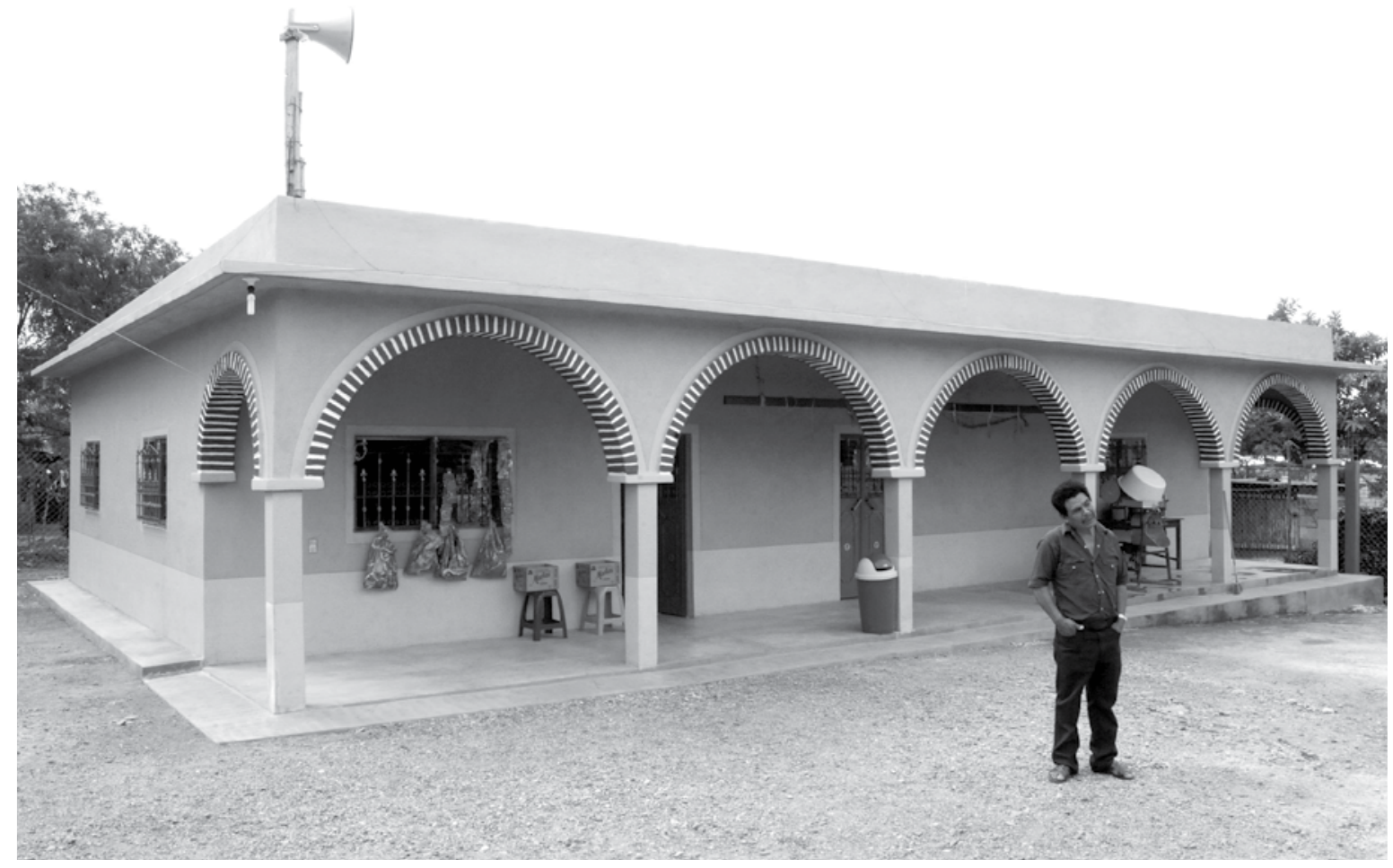

RICARDO RAMIREZ ARRIOLA / www.360gradosfoto.com • La migración implica beneficios para los familiares que permanecen en el lugar de origen, como la inversión de las remesas en la renovación o construcción de viviendas. La Gloria, municipio de La Trinitaria, Chiapas, México, 2009. 
eso construye el vínculo afectivo con el medio, y esto es lo que desencadena una reacción emocional ante la noticia de la construcción de la presa: "cuando empezó este movimiento de la presa, lo primero que te da es mucha tristeza, porque un terreno en donde tú estás viviendo, aprendes a quererlo" (E.Sg.7).

El territorio se convierte en algo más que un espacio físico, representa las raíces, los recuerdos, las relaciones humanas, la privacidad, la identidad:

Aquí era más de sentimientos, no era tanto por lo que nos pudieran dar. Pensábamos en todo, pero más que nada, en lo que nosotros ya hemos vivido, porque nadie puede quitarte tu privacidad, tu vida que a ti te ha costado, si un jarrón, una cosa, tiene tu historia [...] en otro lugar no eres nadie, eres un ave, que pasa y total... Aquí, para nuestra gente aquí, sí somos algo (E.Sg.5).

Es notorio que el territorio no es sólo el espacio físico y cultural, sino que incluye también aspectos de las biografias, como los muertos y los recuerdos, además de las relaciones que hemos mencionado. El apego se fundamenta en una práctica cotidiana que alimenta recuerdos que provocan emociones. Esto hace que el lugar se transforme en algo que no sólo pertenece a las personas, sino del que son parte. El tema de los panteones, por ejemplo, es recurrente en las experiencias que suponen un desplazamiento. En casi todas las culturas, el lugar en el que están enterrados los muertos es sagrado, es un vínculo poderoso entre las personas y el lugar, y presupone una preocupación más ante el posible desplazamiento: "la gente peleaba los muertos, decía: 'los muertos, ¿qué va a pasar con ellos?"” (E.Sg.5).

En el caso de San Gaspar, que presenta una tasa elevada de población migrante a Estados Unidos, el pueblo representa el lugar al cual volver, el espacio familiar en el que se puede recuperar el estilo de vida, las relaciones familiares y de amistad, y la identidad. No tener un lugar al cual volver cuando se desee presupone desarraigo, un sentimiento de abandono y soledad, entre otras consecuencias causadas por la construcción de un embalse.

Todas estas emociones construyen el vínculo con el territorio y juegan un papel muy importante en los proceso cognitivos. Como veremos más adelante, eso contribuye a ver las cosas de manera distinta y a apreciar más lo que se tiene delante.

En este apartado hemos demostrado la importancia de los vínculos entre las personas y el lugar en el que viven, y cómo estos vínculos influyen en las razones que acompañan y legitiman la oposición a la construcción de la presa. Estas reflexiones nos llevan a la segunda parte del análisis, en la que presentaremos algunos procesos no siempre evidentes que, en nuestra opinión, ayudan a comprender los procesos sociales y culturales que desencadena la experiencia del conflicto, y que permiten apreciar la importancia de estas experiencias como laboratorios de nuevas prácticas sociales y políticas.

\section{"ES UNA VIDA, ES UNA HISTORIA, ES TODO"}

Aunque la defensa del territorio fue el principal motivo de implicación, algunos de los entrevistados expresaron que el otro aspecto que les animó a involucrarse en la lucha fue la indignación: "la forma en cómo lo hacen $[\ldots]$ como que te quieren ver la cara de tonto [...], yo creo que éste fue el motivo que más nos caló, que nos hayan querido ver la cara de tontos" (E.Sg.6). La indignación es un sentimiento que se relaciona con la percepción de una situación injusta. Esta emoción, "que comprende una gran cantidad de conceptos, creencias y expectativas [...] es provocada por la creencia de que alguna norma moral ha sido deliberadamente rota y que un daño y sufrimiento han sido infligidos a personas que no lo merecían" (CadenaRoa, 2005: 81). En nuestro caso, averiguamos que la indignación se siente, por ejemplo, ante la imposibilidad de ejercer un derecho que las personas creen 
legítimo, como manifestarse de manera pacífica para expresar disconformidad o dolor, como nos dijo E.Sg.5. Pero también la indignación es resultado de las mentiras reiteradas por el gobierno o por un acto que se percibe como una injusticia. En particular, "la indignación hacia el propio gobierno puede mover particularmente, cuando en ella influye un sentimiento de traición” (Jasper, 2011: 292). Esa decepción que sienten las personas hacia los políticos será el carburante para el cambio en la dimensión política que describiremos.

Además del dolor que provoca la pérdida o la idea de la pérdida del lugar querido, otra emoción que puede influir en la protesta, aunque de modo negativo, es el miedo. El miedo es un sentimiento relacionado con la percepción del riesgo y la amenaza. Para los pobladores de nuestro estudio, el miedo se relacionaba con la preocupación por lo que pudiera pasar en caso de que fuera necesario abandonar el pueblo, que desaparecería bajo las aguas: “yo ni dormía, todos tenían miedo y coraje, miedo que, decíamos: ‘¿dónde vamos a dar? ¿Dónde nos van a llevar?” (E.Sg.13).

El miedo se enfrentó de manera colectiva, por medio de la unión y la solidaridad. En cuanto a la solidaridad desde fuera de la comunidad, los entrevistados comentaron la importancia de conocer experiencias similares, de hablar con personas que habían pasado por lo mismo, como los afectados de Arcediano o "los de Atenco", quienes, con su visita, "inyectaron valor" (E.Sg.5) a la comunidad de San Gaspar. Como veremos en el último apartado de este artículo, la superación del miedo es parte de la transformación que los sujetos experimentan al participar en la protesta.

Entre las emociones relacionadas con la experiencia de la movilización, queremos destacar una que influye en la motivación: el orgullo. Cumple una función relevante en la orientación de la conducta (Reed, 1994) y se relaciona con la alegría y la satisfacción por el logro. El orgullo que la gente siente como consecuencia de haber sabido defender su pueblo se suma a las demás emociones que explican las causas y la intensidad de la movilización, además de fortalecer los vínculos entre los miembros de la comunidad.

La alegría es otra emoción que se produce en la ocurrencia de acontecimientos positivos. En nuestro caso, la alegría se manifestó en el éxito de la movilización y en la participación masiva, lo que dio lugar a una energía emocional (Collins, 2012; Jasper, 2011) que puede alimentar proyectos y luchas futuros. Las emociones relacionadas con experiencias positivas inciden en el cambio porque "comparten la propiedad de ampliar los repertorios de pensamiento y de acción de las personas y de construir reservas de recursos físicos, intelectuales, psicológicos y sociales disponibles para momentos futuros de crisis" (Vecina, 2006: 10). En San Gaspar pudimos observar que la alegría motivó a las personas a seguir adelante y todos reconocen que una de las claves del éxito de su movilización "fue un entusiasmo... la gente ese día se sintió tan apoyada, como dicen, se prendieron los ánimos” (E.Sg.1).

El hecho de que el ambiente positivo y la alegría vivida influyeran también en las relaciones entre las personas, nos conduce a la característica del conflicto como catalizador social. Entre las consecuencias de estas experiencias, está la creación de redes de personas que no imaginaban compartir inquietudes o sueños antes del conflicto, aunque se conocieran.

No podemos dedicar más espacio al papel de las emociones en la movilización porque queremos dar una pincelada de todo el conjunto del análisis. Queremos recordar que hay otras emociones que desempeñaron un papel importante en la movilización, por ejemplo, la esperanza relacionada con la posibilidad de que se consiguiera salvar el pueblo y la rabia hacia los políticos y quienes promovían y defendían la obra. Como observan Regalado y Bañuelos: 
El conflicto social por el acceso, la conservación y la defensa del agua ya es una realidad en México. Como es normal en la disputa, defensa y conservación de los recursos hídricos, el gobierno siempre aparece al lado contrario de los intereses sociales comunitarios y ligado a intereses particulares. El conflicto social aparece en muchas ocasiones debido al autoritarismo con el que el gobierno toma sus decisiones (2006: 183).

El Estado, que posee la legitimidad de actuar para el interés general, promueve de manera unilateral, y sin diálogo con las poblaciones locales, presas y obras hidráulicas que amenazan la existencia de pueblos y comunidades. Eso explica por qué "la resistencia contra las grandes represas es frecuentemente una resistencia contra el Estado" (Martínez, 2004: 168). La experiencia de conflicto entre comunidades locales y Estado se convierte en el detonador del proceso de transformación de conciencia y conducta (Piven y Cloward, 1977) que analizaremos, que se traduce en la metáfora del despertar, utilizada por todos los entrevistados: "ya aprendiste, ya viviste una experiencia, y a lo mejor eso te abrió los ojos, [ya sabes] cómo debes hacer las cosas" (E.Sg.8).

\section{Las luchas por la defensa del territorio como experiencias emancipadoras}

Cable y Degutis hacen hincapié en la importancia de analizar los efectos menos evidentes de las protestas en los niveles individual y colectivo. Afirman que "los resultados relevantes de un movimiento incluyen también aquellas consecuencias involuntarias que favorecen el cambio social" (1997: 123). Estos resultados inesperados de la protesta son las grietas en el sistema de las que habla Holloway (2010). En otras palabras, los "pequeños cambios que se acumulan hasta que el sistema colapse" (Garner, 1977: 408, citado en Cable y Degutis, 1993: 132). Entre ellos incluimos aquellas "consecuencias relacionales, cognitivas y emocionales de la protesta" a las que Della Porta (2008) se refiere con la lucha contra el tren de alta velocidad (TAV) ${ }^{11}$ en Piamonte, Italia. Desde esta perspectiva, la protesta se convierte en un laboratorio cultural que, independientemente de los resultados más evidentes, influye en el cambio social. Para concluir, queremos presentar la trasformación de conciencia y de conducta que hemos analizado en la experiencia de la resistencia contra la presa de San Nicolás, en tres dimensiones: en la relación con el territorio, en la dimensión política y en la biográfica.

Los cambios en la relación con el territorio se han manifestado en la revalorización tanto de sus componentes físicos - el río, los cerros, el campo, etc.- , como culturales y sociales - el pueblo, el estilo de vida, la seguridad, los vínculos afectivos, etc.- -

[He aprendido] a valorar más mi pueblo, quererlo más, y tratar seguir adelante defendiendo lo que al gobierno no le ha costado nada, ni tampoco ha pisado [...]. Sí, nos quedó una experiencia de que a hoy valoramos nuestro pueblito, más que nunca, sabemos que estas son nuestras raíces [...], y que vamos a estar siempre en este pueblito luchando por él y por nosotros, por nuestras tranquilidades, nuestros muertos, por nuestra gente, por ser alguien en la vida. Yo pienso que solamente aquí somos algo (E.Sg.5).

Se comprueba también que como consecuencia del conflicto hay una revalorización de valores, sentimientos y del patrimonio inmaterial, como recuerdos, raíces, etc. Mientras las administraciones siguen la lógica costo-beneficio puramente materialista sobre la que se basa, por ejemplo, la práctica de las expropiaciones, las personas de las comunidades afectadas rechazan esta lógica, reconocen el valor 
sentimental y humano de la pérdida, se replantean el valor de las cosas y admiten la inconmensurabilidad de esos valores. En esta línea, apreciamos un cambio significativo en la definición de progreso, puesto que las obras que amenazaban el territorio eran presentadas por los gobiernos como ejemplos de progreso, por lo que se estigmatizaba a quienes se oponían por ir en contra del progreso y del interés general:

El progreso muchas veces no representa de veras un beneficio, un bien para el pueblo, a veces más bien eso llena los bolsillos o incrementa los negocios o le da beneficios a quien los dirige los proyectos [...], entonces, a veces, decimos: “¿para quién fue el progreso realmente?" (E.Sg.1).

En relación con la sensibilidad ambiental, no percibimos un cambio importante. Las personas que tenían una sensibilidad antes del conflicto la mantienen, aunque se incrementó la necesidad de transmitir esa sensibilidad a las nuevas generaciones, entre otras razones, como estrategia defensiva ante ataques futuros. Este último elemento no tiene nada de extraño porque estas experiencias son conflictos sociales y políticos antes que ambientales. Surgen de la resistencia contra el Estado para la supervivencia, en lugares que además son periféricos, en relación con los centros económicos y de poder, y por esa razón pueden ser sacrificados para llevar riqueza a otros lugares.

En el análisis de la dimensión política, observamos elementos interesantes. Primero, el empoderamiento de las personas involucradas en estas experiencias ha hecho que se conviertan en nuevos sujetos políticos, con capacidad de oponerse a lo que no creen justo y de tener derecho a vivir según sus principios y necesidades. Ese empoderamiento se ve en la legitimación de la lucha y de la protesta como medio para defenderse y conseguir derecho, y en la pérdida del miedo a la autoridad, sea de los políticos o de los policías: "yo siento que desde entonces no le tengo miedo ni al gobierno, ni a nadie, son humanos también y también tienen miedo igual que la gente" (E.Sg.4).

El descrédito y la desconfianza hacia la clase política y la política institucional en general son comunes en las experiencias de lucha por la defensa del territorio, se acompañan por la revalorización de la fuerza de los sujetos y la toma de conciencia de la posibilidad de vivir de manera digna en sus territorios sin la intervención del Estado. Estos sentimientos se reflejan en la reelaboración de la idea de democracia que, como concepto abstracto, es percibida positivamente, aunque todos reconozcan que en la práctica estamos lejos de conseguirla:

Desgraciadamente eso a mí me hace dudar de la democracia en México todavía, para que alcance su totalidad creo que faltan años y espero que no sean necesarias revoluciones $u$ otros movimientos para hacer entender a la clase política que ya queremos ver resultados reales, transformaciones, acuerdos, que en verdad mejoren la vida de los pueblos, porque hay con qué mejorarla (E.Sg.1).

El empoderamiento, es decir, el "poder de" cambiar las cosas, se fortalece por el éxito del conflicto. Las personas entrevistadas reconocen la legitimidad de la lucha no sólo para conseguir sus objetivos, sino también para defender su dignidad. Las personas empoderadas no sólo consideran la lucha como una vía legítima para defenderse y actuar contra las injusticias, sino que también reconocen la importancia de la unión para conseguir sus objetivos:

Fue cuando nosotros sentimos que ante nadie, o sea ante nadie tenía valor lo que nosotros estábamos defendiendo, era una lucha de la comunidad nada más, sin ninguna autoridad ni ningún partido político y sin ningún apoyo, ni religioso, ni de ningún lado, era rifárnosla nosotros solos, y así nos aventamos, se unió la comunidad, y salimos adelante (E.Sg.9). 


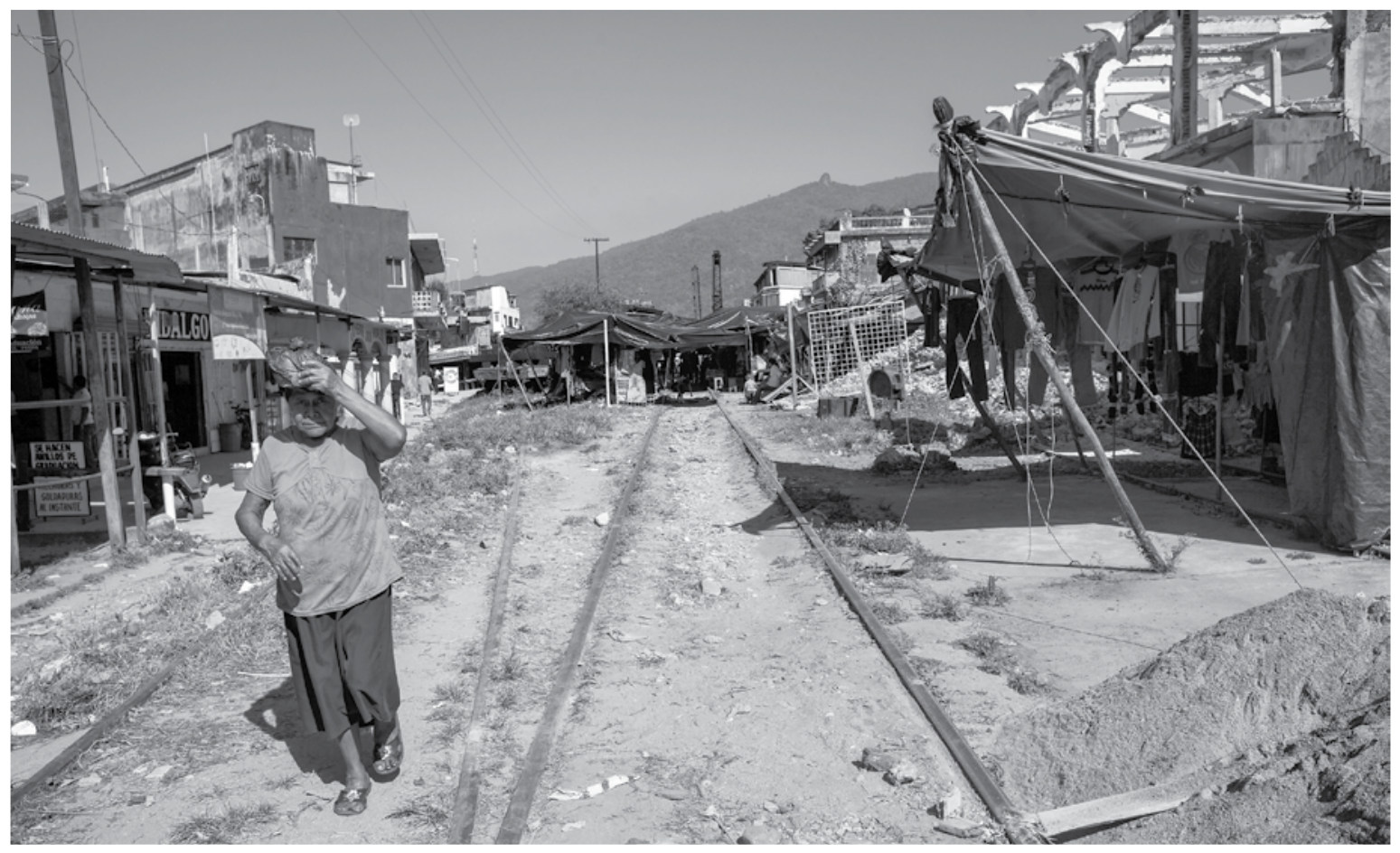

PROMEteo LuCERo - La estructura de desigualdad del proceso migratorio da lugar a flujos de personas de países pobres hacia países ricos o desde economías muy empobrecidas hacia otras menos empobrecidas. Huixtla, municipio chiapaneco en el que se han asentado numerosas familias centroamericanas, diciembre de 2014

Piven y Cloward (1977) hablan de la emergencia de un "nuevo sentimiento de eficacia", que se produce en las personas que de ordinario se consideran políticamente impotentes y que a raíz de la experiencia de protesta comienzan a creer en su capacidad para cambiar las cosas. Ese empoderamiento de los implicados en el conflicto se manifiesta en cambios individuales y colectivos, en la esfera personal —empoderamiento psicológico-y en la política —empoderamiento político- - Respecto a la dimensión individual, quienes participaron en estas resistencias han visto crecer su autoestima, han superado el miedo a hablar en público o a actuar públicamente:

Fue como un momento que me hizo revalorarme, o sea descubrir quizá capacidades que no había desarrollado, o sea como que me revaloré a mí mismo [...]. No me imaginaba el cambio que iba a traer esto en mí realmente [...], descubrí que yo podía también, podía tener la capacidad de hablar [...], fueron momentos que sí despertaron en mí otro tipo de persona que no era yo (E.Sg.1).

La experiencia de lucha hace que se creen nuevos vínculos entre las personas que no sabían que compartían necesidades o visiones del mundo, y se fortalecen las relaciones de la comunidad o de los grupos que comparten la vivencia de la protesta.

Percibimos también un cambio en los prejuicios hacia determinados colectivos, como los ecologistas, y en general hacia quien lucha. La práctica de la resistencia implica conocer en profundidad a personas que pertenecen a estos colectivos, confrontarse y encontrarse en el lugar de los que tienen 
que luchar para defender su territorio, a quienes no comprendían porque sólo los habían visto en los medios de comunicación, es decir, con las lentes de la ideología dominante: "muchas veces que ves en la televisión manifestaciones y dices: 'ah, gente borlotera, y esto y el otro'. Yo decía primero y ya hoy no, hoy digo: 'están bien, porque están defendiendo sus causas', y es lo mismo que nosotros andábamos haciendo" (E.Sg.9).

En la población queda la desconfianza hacia las instituciones, la cual, unida al empoderamiento y a la conciencia de que si se unen pueden vencer hasta al gobierno, lleva a la gente a estar pendiente de lo que pasa en su territorio. Los habitantes de San Gaspar han conseguido un control exhaustivo del territorio y después de terminado el conflicto siguen atentos y alerta. Todos los entrevistados afirman que aunque haya sido una experiencia muy dura, la gente está lista para defenderse en cualquier momento que se presente una amenaza. Esperamos no tener que comprobarlo, porque significaría que la vida del pueblo estaría en peligro.

El empoderamiento se manifiesta en nuevas prácticas que surgen a raíz de la experiencia de lucha y que reflejan parte del cambio analizado. Por ejemplo, el trabajo cotidiano de algunas personas del pueblo, como la sensibilización ambiental con los niños en la escuela o el catecismo, intenta incidir en las costumbres del pueblo en materia ambiental. Otros momentos importantes son las fiestas de los pueblos. El 31 de mayo de 2005 se anunció a la población de San Gaspar que se desestimaba el proyecto. Desde entonces, cada año, durante la fiesta en honor de su patrona, María Auxiliadora, se recuerda este evento de la historia local, que ha sido un parteaguas para los que lo vivieron. Otro ejemplo es la protesta de los habitantes de San Gaspar que fueron a Jalostotitlán, la cabecera municipal de la que dependen, por primera vez en 2007, para exigir la elección del delegado en el ayuntamiento, por lo regular elegido por el alcalde:
Sucedió el pasado martes. Un centenar y medio de pobladores de San Gaspar de los Reyes estuvieron [sic] en manifestación en el palacio municipal. El alcalde de Jalos recibió a una comisión en dos ocasiones y se logró un acuerdo: irían con el señor Evaristo Tiscareño para convencerlo de que el delegado en esa población debería salir de una consulta con el pueblo (Casillas, 2007).

El análisis de la dimensión cultural del conflicto, que comprende creencias cognitivas, respuestas emocionales y evaluaciones morales (Jasper, 1997), pone en evidencia la transformación de conciencia y de conducta que viven las personas que defienden su territorio, que convierte estas experiencias de lucha y resistencia en experiencias emancipadoras.

\section{Conclusiones}

El análisis de la resistencia contra la construcción de la presa de San Nicolás en Los Altos de Jalisco confirma la hipótesis de que la experiencia del conflicto produce una transformación de conciencia y conducta que se aprecia en el nivel micro. Este cambio se manifiesta en varias dimensiones: la relación con el territorio cuyo vínculo se refuerza, la toma de conciencia, la identidad, las relaciones en la comunidad y la dimensión política. Los cambios suelen ser tanto personales como colectivos, y se reflejan en nuevas prácticas que surgen gracias a los vínculos entre las personas creados durante el conflicto, al conocimiento aprendido y a la conciencia de que es posible cambiar.

El estudio de caso también permitió constatar la solidez de nuestras hipótesis, entre las que destaca la importancia de las emociones en el estudio de la movilización. Apreciamos la importancia de las emociones en la motivación a la acción relacionadas tanto con el apego al territorio como con la felicidad de ver al pueblo levantado contra el proyecto, y la vinculación de las emociones con el impulso a la acción. 
En relación con el apego al territorio, observamos la intensidad emocional con la que se vincularon los entrevistados a su entorno natural, y el sentimiento de apropiación hacia éste. Un elemento interesante es que las personas se reapropian del territorio y lo consideran algo que les pertenece y que quieren, sentimiento que tendría que ser revaluado por ser un camino útil para llegar a desarrollar una conciencia hacia el medio ambiente, puesto que se protege lo que se ama.

Estos primeros resultados ponen de manifiesto la necesidad de seguir analizando la dimensión cultural de las experiencias de lucha por el agua y la defensa del territorio, por ejemplo, y excavar más tanto en el papel de las emociones como factores explicativos de estos procesos como en la relación entre el ser humano y el territorio en el que vive.

Creemos que el estudio en profundidad de estos procesos, desde la perspectiva de la gente que lucha, permite conocer una faceta de los procesos de resistencia locales que hasta ahora había estado escondida a la mirada de los investigadores, ya que las personas "en el proceso de decir ‘ ‘No!', desarrollan formas de autodeterminación o articulan conceptos alternativos de cómo debería ser el mundo" (Holloway, 2009: 17). D

\section{Bibliografía}

Cable, Sherry y Beth Degutis, 1997, “Movement Outcomes and Dimensions of Social Change: The Multiple Effects of Local Mobilization”, en Current Sociology, vol. 45, núm. 3, pp. 121-135.

Cabrales Barajas, Luis Felipe, 1993, "El río Verde: agua para Guadalajara”, en Carta Económica Regional, núm. 19, pp. 3-15.

Cadena-Roa, Jorge, 2005, "Strategic Framing, Emotions, and Superbarrio-Mexico City's Masked Crusader", en Hank Johnston y John A. Noakes (eds.), Frames of Protest: Social Movements and the Framing Perspective, Rowman \& Littlefield, Lanham, pp. 69-86.

Casillas Báez, Miguel Ángel, 1999, “Guadalajara y la región alteña: la preocupación por el abasto de agua”, en Cándido González Pérez (coord.), Tercer Simposium Los Altos a Fin de Siglo, Memorias, Universidad de Guadalajara, Guadalajara, pp. 95-130.

—_, 2002, La tercera revolución del agua, sociedad y medioambiente en Los Altos de Jalisco, El Colegio de Jalisco/Comisión Estatal de Agua y Saneamiento, Zapopan.

—_, 2007, "Nombramiento para 9 horas: San Gaspar de los Reyes eligió a Martín Quezada”, en Los Altos, 5 de enero, p. 1.

Collins, Randall, 2012, "C-escalation and D-escalation: A Theory of the Time-Dynamics of Conflict", en American Sociological Review, vol. 77, núm. 1, pp. 1-20.

Della Porta, Donatella, 2008, "Eventful Protest, Global Conflicts”, en Distinktion: Journal of Social Theory, vol. 9 núm. 2, pp. 27-56.

Devine-Wright, Patrick, 2009, "Rethinking NIMBYism: The Role of Place Attachment and Place Identity in Explaining Place-Protective Action", en Journal of Community \& Applied Social Psychology, vol. 19, núm. 6, pp. 426-441.

Fedi, Angela y Terri Mannarini (eds.), 2008, Oltre I/ Nimby. La Dimensione Psico-Sociale della Protesta Contro le Opere Sgradite, FrancoAngeli, Milán. Giuliani, Maria Vittoria, 2004, “Teoria dell'attaccamento ai luoghi”, en Mirilia Bonnes, Marino Bonaiuto y Raffaello Terence Lee (eds.), Teorie in pratica per la psicologia ambientale, Cortina Editore, Milán, pp. 191-240.

Goleman, Daniel, 1996, La inteligencia emocional, Kairós, Barcelona.

Goodwin, Jeff, James M. Jasper y Francesca Polletta (eds.), 2001, Passionate Politics: Emotions in Social Movements, University of Chicago Press, Chicago.

Gould, Deborah B., 2004, "Passionate Political Processes: Bringing Emotions Back into the Study of Social Movements", en Jeff Goodwin y James M. Jasper (eds.), Rethinking Social Movements: Structure, Meaning and Emotion, Rowman \& Littlefield, Lanham, pp. 155-175.

Hernández López, José de Jesús y Miguel Ángel Casillas Báez, 2006, “Impacto social e intereses económico-políticos en la formación de megacuencas. El caso de la presa de San Nicolás, Jalisco", ponencia presentada en el Tercer Coloquio Internacional de Temas Jaliscienses, Ayuntamiento de Lagos de Moreno, Guadalajara.

__ , 2008, "La presa que se llenó de engaños: el caso de San Nicolás, Jalisco. Respuesta regional a los proyectos estatales de trasvases", en Relaciones, vol. 29, núm. 116, pp. 23-62.

Hidalgo Villodres, María del Carmen, 1998, Apego al lugar: ámbitos, dimensiones y estilos, tesis de doctorado, Universidad de la Laguna, Tenerife. Holloway, John, 2009, “Teoría volcánica”, en John Holloway, Fernando Matamoros y Sergio Tischler (eds.), Pensar a contrapelo: movimientos sociales y reflexión crítica, Benemérita Universidad Autónoma de Puebla/Bajo Tierra Ediciones/Sísifo Ediciones, Puebla, pp. 15-29. 
- 2010, Crack Capitalism, Pluto Press, Londres.

, 2011, Agrietar el capitalismo. El hacer contra el trabajo, Herramienta, Buenos Aires.

Jasper, James M., 1997, The Art of Moral Protest: Culture, Biography, and Creativity in Social Movements, University of Chicago Press, Chicago.

-, 1998, "The Emotions of Protest: Affective and Reactive Emotions in and around Social Movements", en Sociological Forum, vol. 13, núm. 3, pp. 397-424.

, 2011, "Emotion and Social Movements: Twenty Years of Theory and Research", en Annual Review of Sociology, vol. 37, pp. 285-303.

, 2014, "Constructing Indignation: Anger Dynamics in Protest Movements", en Emotion Review, vol. 6, núm. 3, pp. 208-213.

Krauss, Celene, 1993, "Women and Toxic Waste Protests: Race, Class and Gender as Resources of Resistance”, en Qualitative Sociology, vol. 16, núm. 3, pp. 247-262.

Low, Setha M. e Irwin Altman, 1992, Place Attachment, Plenum Press, Nueva York.

Mairal Buil, Gaspar, 1993, “'Perder el pueblo’. Antropología aplicada y política hidráulica”, en Revista de Antropología Social, vol. 2, pp. 185-237.

Mairal Buil, Gaspar, José A. Bergua y Esther Puyal E., 1997, Agua, tierra, riesgo y supervivencia: un estudio antropológico sobre el impacto socio-cultural derivado de la regulación del río Esera, Prensas Universitarias de Zaragoza, Zaragoza.

Manzo, Lynne C. y Patrick Devine-Wright (eds.), 2014, Place Attachment: Advances in Theory, Methods and Applications, Routledge, Nueva York. Martínez Alier, Joan, 2004, El ecologismo de los pobres. Conflictos ambientales y lenguajes de valoración, Icaria, Barcelona.

McCully, Patrick, 2004, Ríos silenciados. Ecología y politica de las grandes represas, Proteger Ediciones, Buenos Aires.

Pearce, Fred, 1992, The Dammed: Rivers, Dams and The Coming World Water Crisis, The Bodley Head, Londres.

Piven, Frances Fox y Richard A. Cloward, 1977, Poor People's Movements. Why They Succeed, How They Fail, Pantheon Books, Nueva York.

Pleyers, Geoffrey, 2009, "Autonomías locales y subjetividades en contra del neoliberalismo: hacia un nuevo paradigma para entender los movimientos sociales", en Francis Mestries Benquet, Geoffrey Pleyers y Sergio Zermeño (eds.), Los movimientos sociales: de lo local a lo global, Anthropos, Barcelona, pp. 129-156.

Poma, Alice, 2013, Conflictos ambientales y cambio cultural. Un análisis desde la perspectiva de los afectados, tesis de doctorado, Universidad Pablo de Olavide, Sevilla.

Reed, Jean Pierre, 1994, "Emotions in Context: Revolutionary Accelerators, Hope, Moral Outrage, and Other Emotions in the Making of Nicaragua's Revolution", en Theory and Society, vol. 33, núm. 6, pp. 653-703.

Regalado, Jorge, 2012 "Notas deshilvanadas sobre otra epistemología”, en Jorge Regalado et al. (eds.), Hacer política para un porvenir más allá del capitalismo, Grietas Editores, Guadalajara, pp. 167-181.

Regalado, Jorge y Luis H. Bañuelos Medina, 2006, "La gestión del agua en Jalisco", Jesús Raúl Navarro García y Jorge Regalado Santillán (eds.), El debate del agua en Jalisco y Andalucía, Escuela de Estudios Hispanoamericanos-Consejo Superior de Investigaciones Científicas/Asociación Cultural la Otra Andalucía, Sevilla, pp. 153-184.

Scott, James C., 2000, Los dominados y el arte de la resistencia, Era, México.

Thompson, Paul, 1993, "Historias de vida en el análisis de cambio social”, en José Miguel Marinas y Cristina Santamarina (eds.), La historia oral: métodos y experiencias, Debate, Madrid, pp. 65-80.

Tomé Martín, Pedro y Andrés Fábregas Puig, 1999, Entre mundos. Procesos interculturales entre México y España, Diputación Provincial de Ávila/El Colegio de Jalisco, Zapopan.

Vecina Jiménez, María Luisa, 2006, “Emociones positivas”, en Papeles del Psicólogo, vol. 27, núm. 1, pp. 9-17.

Ward, Colin, 2003, Acqua e comunitá, Elèuthera, Milán.

\section{Entrevistas}

E.Sg.1, hombre, miembro del Comité Pro San Gaspar, noviembre de 2010.

E.Sg.4, hombre, miembro del Comité Pro San Gaspar, noviembre de 2010.

E.Sg.5, mujer, miembro del Comité Pro San Gaspar, noviembre de 2010.

E.Sg.5b, hombre, habitante de San Gaspar, noviembre de 2010.

E.Sg.6, mujer, miembro del Comité Pro San Gaspar, enero de 2011.

E.Sg.7, hombre, afectado de Teocaltiche, enero de 2011.

E.Sg.8, mujer, miembro del Comité Pro San Gaspar, enero de 2011.

E.Sg.9, mujer, miembro del Comité Pro San Gaspar, enero de 2011.

E.Sg.10, mujer, habitante de San Gaspar, enero de 2011.

E.Sg.13, mujer, habitante de San Gaspar, enero de 2011. 